Supporting Information

\title{
Z-DNA as a Touchstone for Additive Empirical Force Fields and a Refinement of the Alpha/Gamma DNA torsions for AMBER
}

Marie Zgarbová, ${ }^{1}$ Jiř́ Š Soner, ${ }^{2}$ Petr Jurečka ${ }^{1 *}$

${ }^{1}$ Department of Physical Chemistry, Faculty of Science, Palacky University, 17. listopadu 12, 77146 Olomouc, Czech Republic

${ }^{2}$ Institute of Biophysics, Academy of Sciences of the Czech Republic, Královopolská 135, 61265

Brno, Czech Republic

*e-mail: petr.jurecka@upol.cz

Table S1. OL21 $\alpha / \gamma$ parameters.

\begin{tabular}{|c|c|c|c|c|}
\hline & Torsion & $n$ & $\mathrm{~V}_{\mathrm{n}} / 2[\mathrm{kcal} / \mathrm{mol}]$ & $\gamma[\operatorname{deg}]$ \\
\hline \multirow{6}{*}{$\alpha$} & \multirow{6}{*}{$\begin{array}{l}\text { C5'-OS-P-OS } \\
(C J-O S-P-O S)^{\text {a) }}\end{array}$} & 1 & 0.3164 & 102.1 \\
\hline & & 2 & 0.6301 & 359.7 \\
\hline & & 3 & 0.2412 & 299.9 \\
\hline & & 4 & 0.1626 & 32.08 \\
\hline & & 5 & 0.0975 & 303.6 \\
\hline & & 6 & 0.0433 & 19.49 \\
\hline \multirow{6}{*}{$\gamma$} & & 1 & 0.4467 & 166.5 \\
\hline & $\mathrm{C} 3^{\prime}-\mathrm{C}^{\prime}-\mathrm{C}^{\prime}-\mathrm{OS}$ & 2 & 0.3842 & 245.8 \\
\hline & $(\mathrm{C7}-\mathrm{CT}-\mathrm{CJ}-\mathrm{OS})^{\mathrm{a}}$ & 3 & 0.7514 & 344.0 \\
\hline & $\mathrm{C}^{\prime}-\mathrm{C}^{\prime}-\mathrm{C}^{\prime}-\mathrm{OH}$ & 4 & 0.1394 & 276.0 \\
\hline & $(\mathrm{C7}-\mathrm{CT}-\mathrm{CJ}-\mathrm{OH})^{\mathrm{a}}$ & 5 & 0.0436 & 305.3 \\
\hline & & 6 & 0.0384 & 141.2 \\
\hline
\end{tabular}

a) C7 and CJ are atom types of the OL15 force field, previously CT type. 
Table S2. Base pair and base pair step parameters and other duplex characteristics for DickersonDrew dodecamer. Simulation in $0.15 \mathrm{M} \mathrm{KCl}$ and SPC/E water, flat well restraints at both ends. Backbone angles were averaged over the canonical region only; the two terminal base pairs at each end were excluded from the analysis.

\begin{tabular}{|c|c|c|c|c|}
\hline & X-RAY & OL15 & bsc1 & OL21 \\
\hline$\alpha / \operatorname{deg}$ & 298.7 & 290.5 & 288.3 & 294.9 \\
\hline$\beta / \operatorname{deg}$ & 176.6 & 180.4 & 176.6 & 180.7 \\
\hline$\gamma /$ deg & 52.6 & 53.6 & 54.3 & 50.2 \\
\hline$\delta /$ deg & 122.4 & 133.0 & 135.0 & 133.5 \\
\hline$\varepsilon /$ deg & 180.1 & 181.7 & 188.1 & 181.3 \\
\hline$\zeta /$ deg & 268.6 & 267.3 & 265.9 & 266.7 \\
\hline$\chi /$ deg & 247.6 & 252.9 & 249.0 & 253.3 \\
\hline $\mathrm{P} / \mathrm{deg}$ & 130.1 & 150.6 & 150.2 & 151.7 \\
\hline 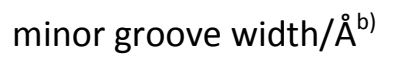 & 10.6 & 11.3 & 11.3 & 11.1 \\
\hline major groove width $/ \AA^{b}$ ) & 17.9 & 17.8 & 18.3 & 17.9 \\
\hline shift/Å & 0.0 & 0.0 & 0.0 & 0.0 \\
\hline slide/Å & 0.0 & 0.0 & -0.3 & 0.0 \\
\hline rise/Å & 3.3 & 3.3 & 3.3 & 3.3 \\
\hline tilt/deg & -0.2 & 0.0 & 0.0 & 0.1 \\
\hline roll/deg & 2.0 & 2.2 & 1.9 & 1.9 \\
\hline twist/deg & 33.6 & 35.2 & 34.3 & 35.2 \\
\hline shear/Å & 0.0 & 0.0 & 0.0 & 0.0 \\
\hline buckle/deg & 0.9 & 0.0 & 0.1 & 0.1 \\
\hline stretch/Å & -0.1 & 0.0 & 0.0 & 0.0 \\
\hline propeller/deg & -11.8 & -11.6 & -9.3 & -11.7 \\
\hline stagger/Å & 0.1 & 0.0 & 0.1 & 0.0 \\
\hline opening/deg & 2.1 & -0.4 & -0.8 & -0.2 \\
\hline x-displacement/Å & -0.5 & -0.4 & -1.0 & -0.4 \\
\hline y-displacement/Å & -0.1 & 0.0 & 0.0 & 0.0 \\
\hline $\mathrm{h}$ rise/deg & 3.3 & 3.3 & 3.2 & 3.3 \\
\hline incl/deg & 4.3 & 4.1 & 4.1 & 3.5 \\
\hline tip/deg & 0.5 & 0.0 & 0.0 & -0.1 \\
\hline $\mathrm{h}$ twist/deg & 34.0 & 36.1 & 35.2 & 36.1 \\
\hline
\end{tabular}


a) Reference values (except for groove widths) taken from Ref. 1 of this work (average over 5 Xray structures).

b) Groove widths measured by cpptraj. 
Figure S1. Base pair step (left) and base pair parameters (right) of Dickerson-Drew dodecamer. Simulation in $0.15 \mathrm{M} \mathrm{KCl}$ and SPC/E water, flat well restraints at both ends.
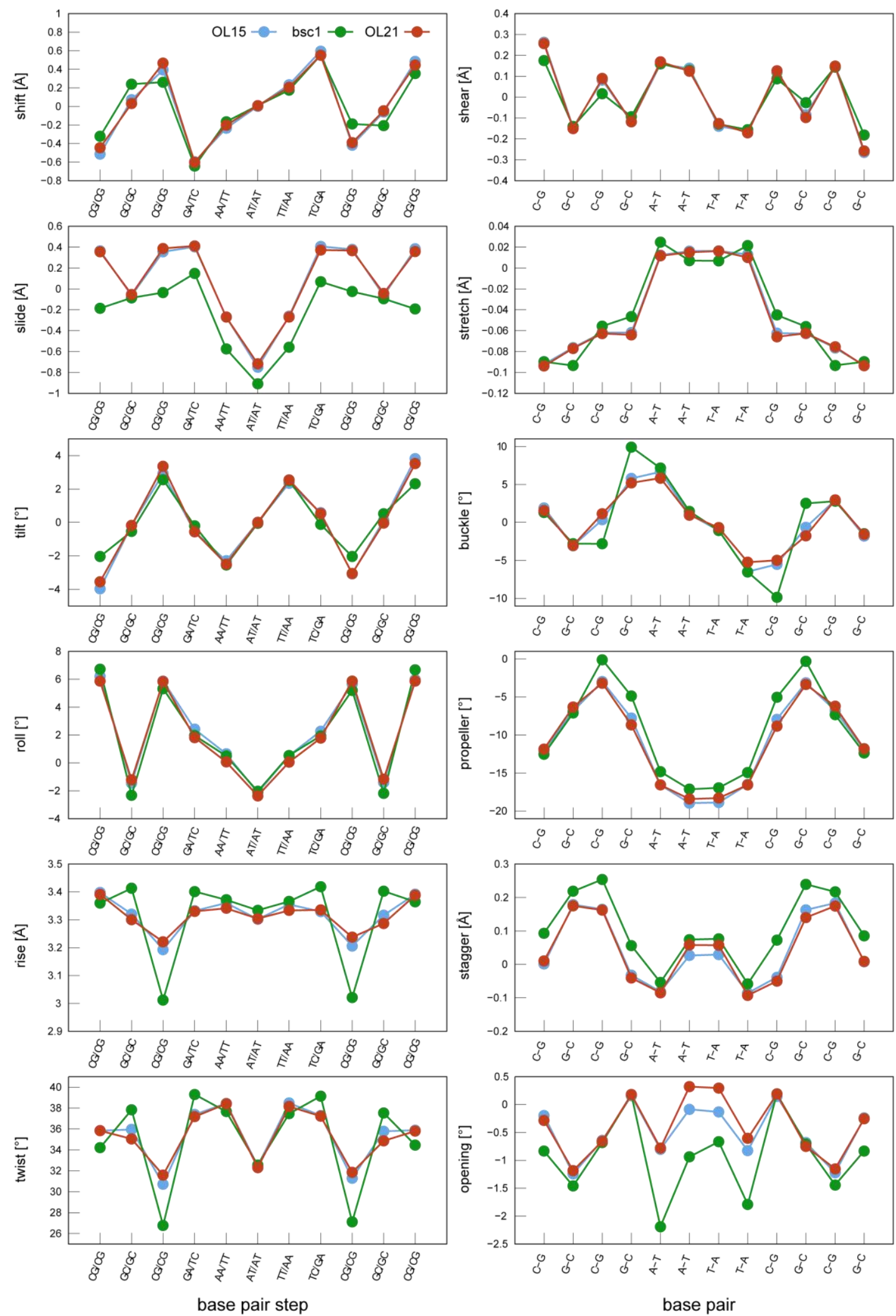
Figure S2. Histograms of the backbone angles, glycosidic angle and sugar pucker for DickersonDrew dodecamer. Unrestrained simulation in $0.15 \mathrm{M} \mathrm{KCl}$ and SPC/E water, the two terminal base pairs at each end were excluded from the analysis.
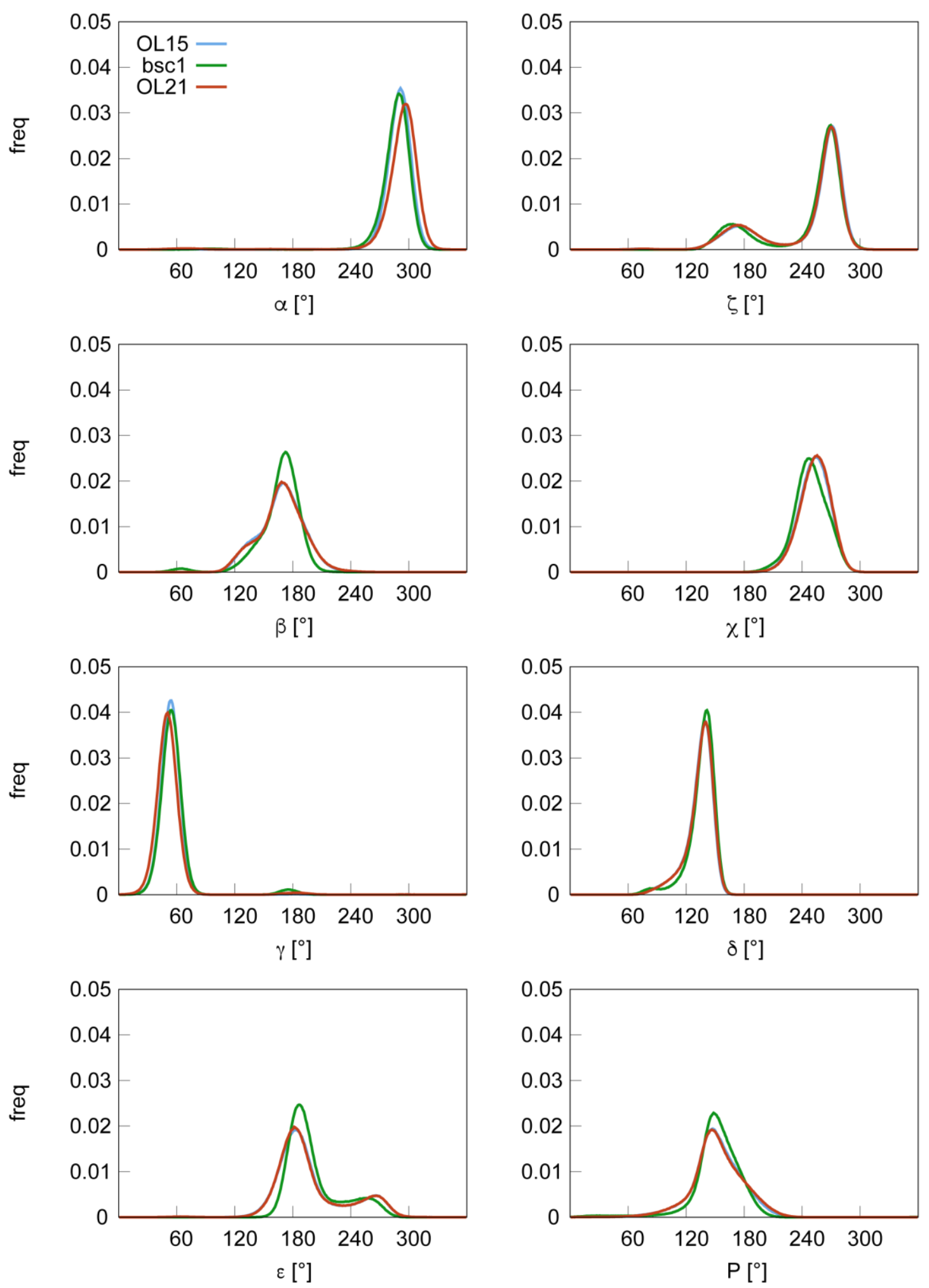
Figure S3. RMSD of Dickerson-Drew dodecamer stem calculated for all atoms against the 1BNA structure (mass weighted). Unrestrained simulation in $0.15 \mathrm{M} \mathrm{KCl}$ and SPC/E water, the two terminal base pairs at each end were excluded from the analysis.

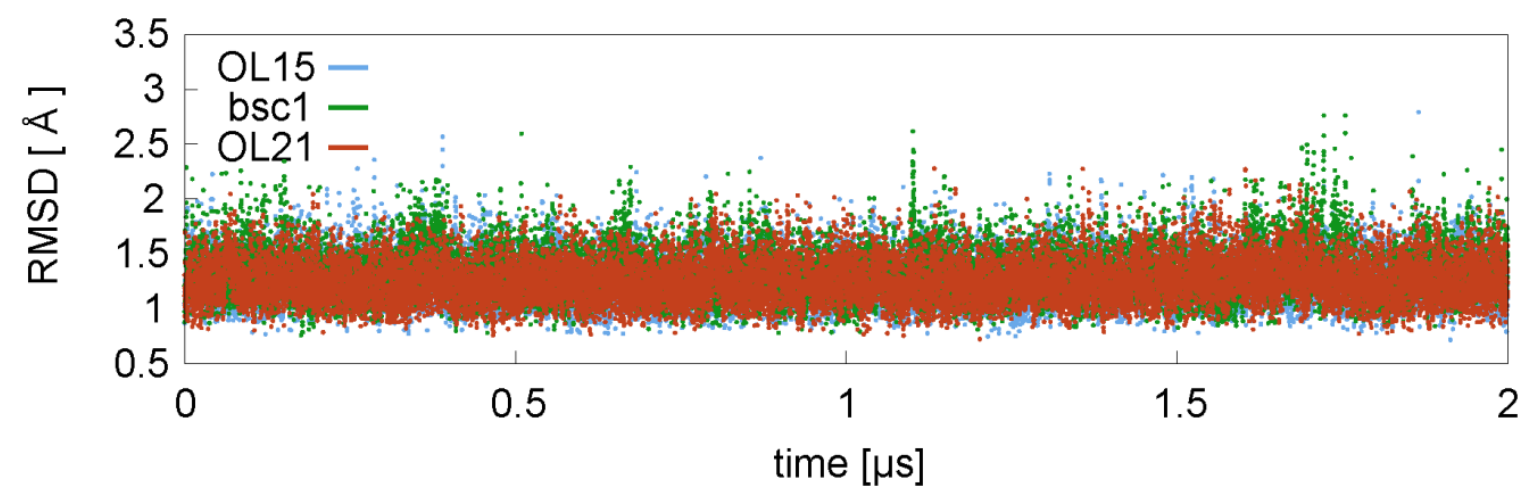

Figure S4. BII percentage values for individual base pair steps in the Dickerson-Drew dodecamer. Simulation in $0.15 \mathrm{M} \mathrm{KCl}$ and SPC/E water, flat well restraints at both ends.

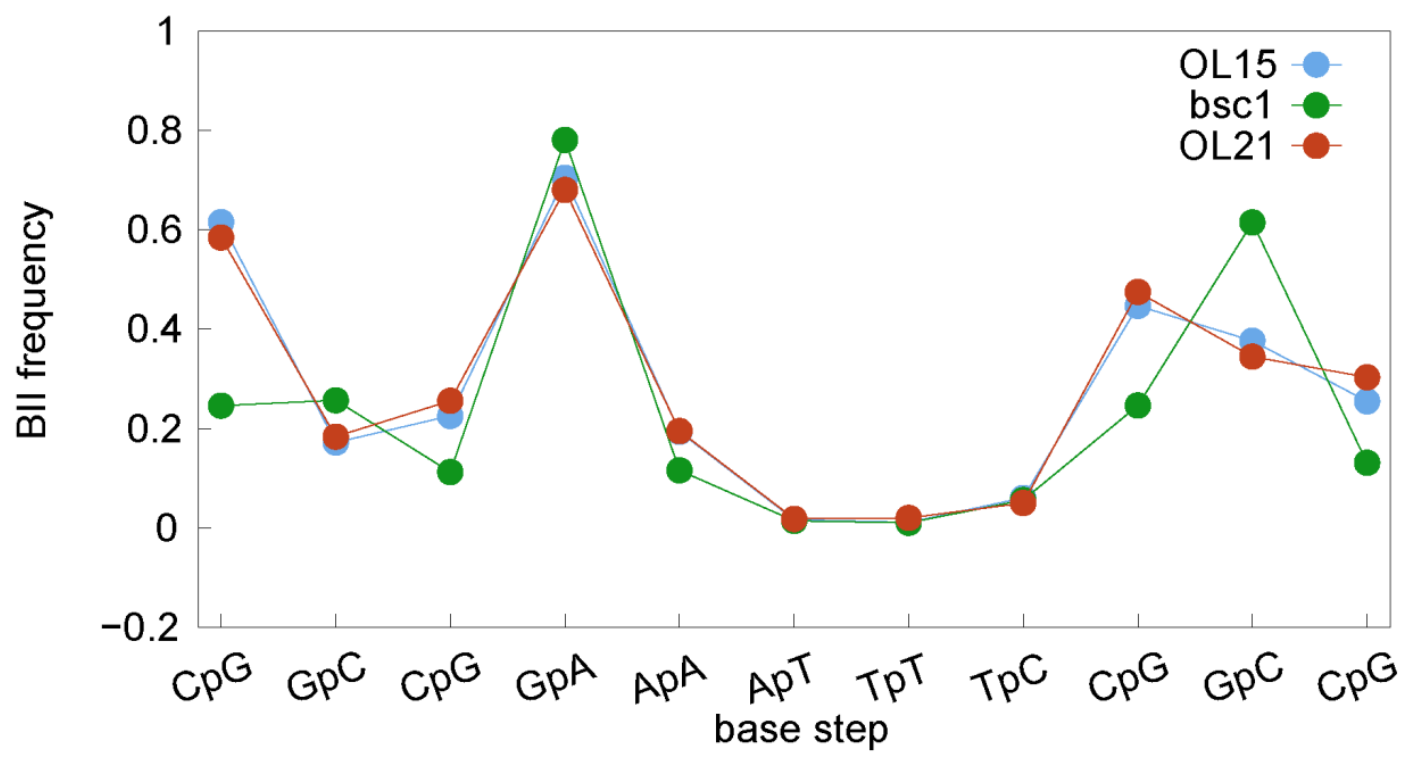


Figure S5. Time evolution of backbone angles $\varepsilon, \zeta$ and $\beta$ in the OL21 $\alpha / \gamma$ simulation of Dickerson-Drew dodecamer. Simulation in $0.15 \mathrm{M} \mathrm{KCl}$ and SPC/E water, flat well restraints at both ends.

OL21
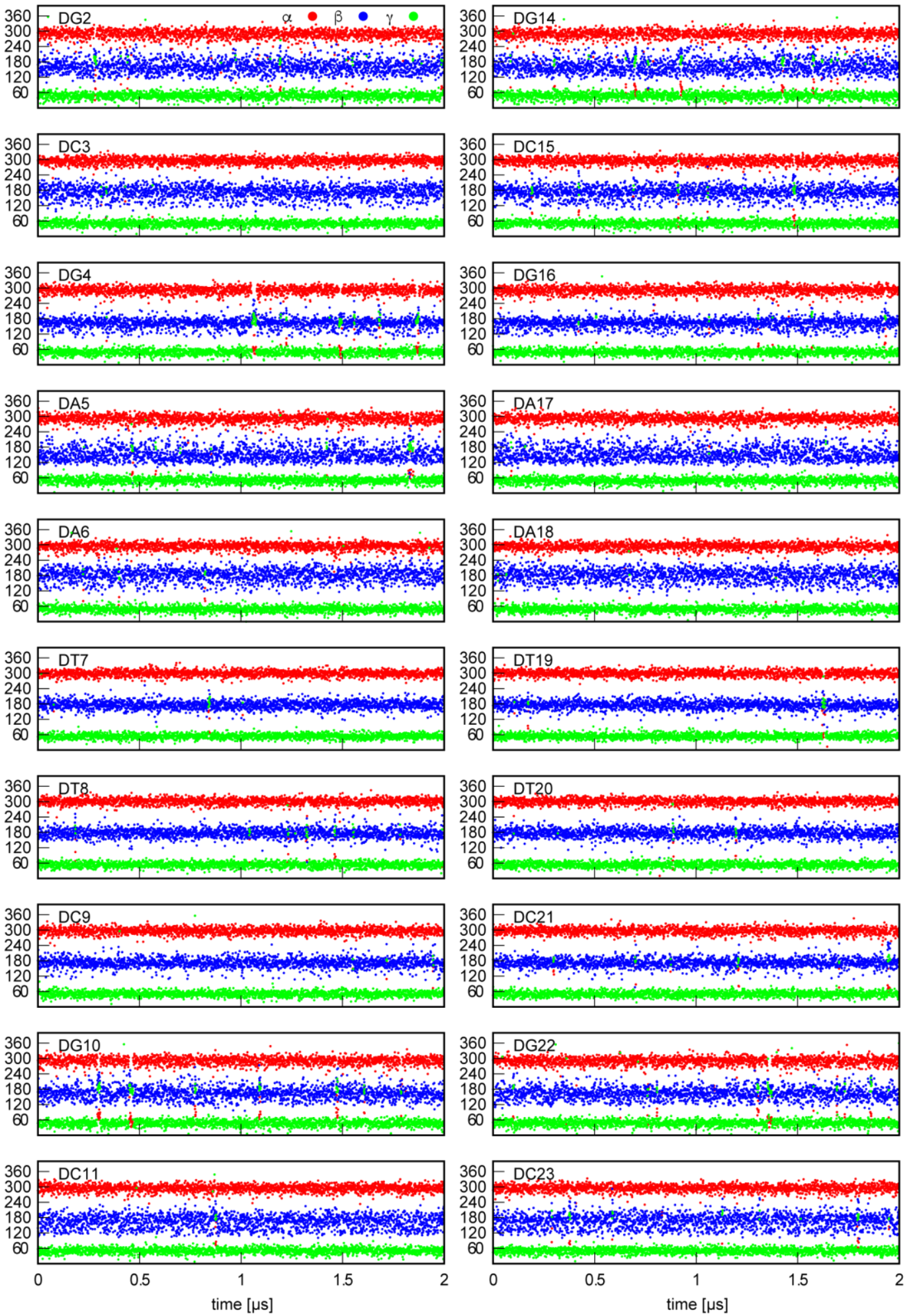
Figure S6. Time evolution of backbone angles $\varepsilon, \zeta$ and $\beta$ in the OL15 $\alpha / \gamma$ simulation of Dickerson-Drew dodecamer. Simulation in $0.15 \mathrm{M} \mathrm{KCl}$ and SPC/E water, flat well restraints at both ends.

OL15
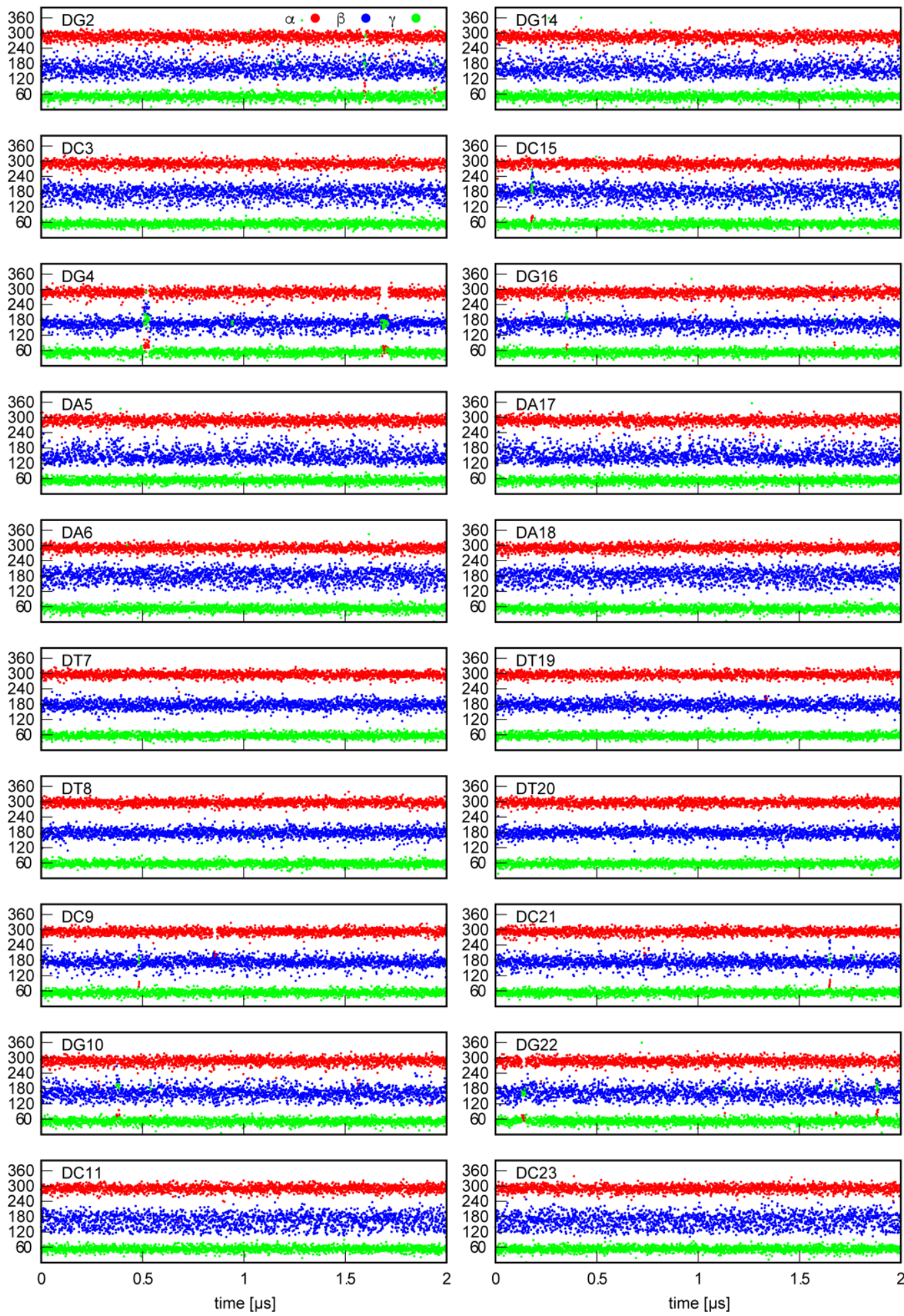
Figure S7. Time evolution of backbone angles $\varepsilon, \zeta$ and $\beta$ in the bsc1 simulation of DickersonDrew dodecamer. Simulation in $0.15 \mathrm{M} \mathrm{KCl}$ and SPC/E water, flat well restraints at both ends. bsc1
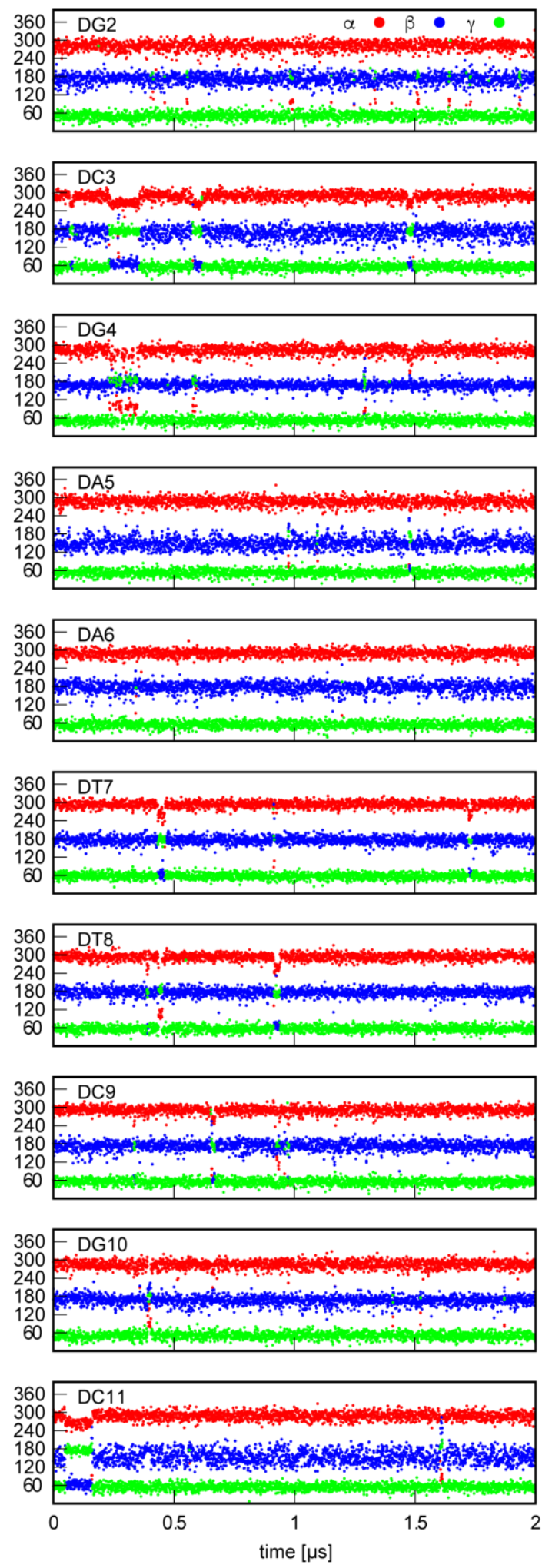
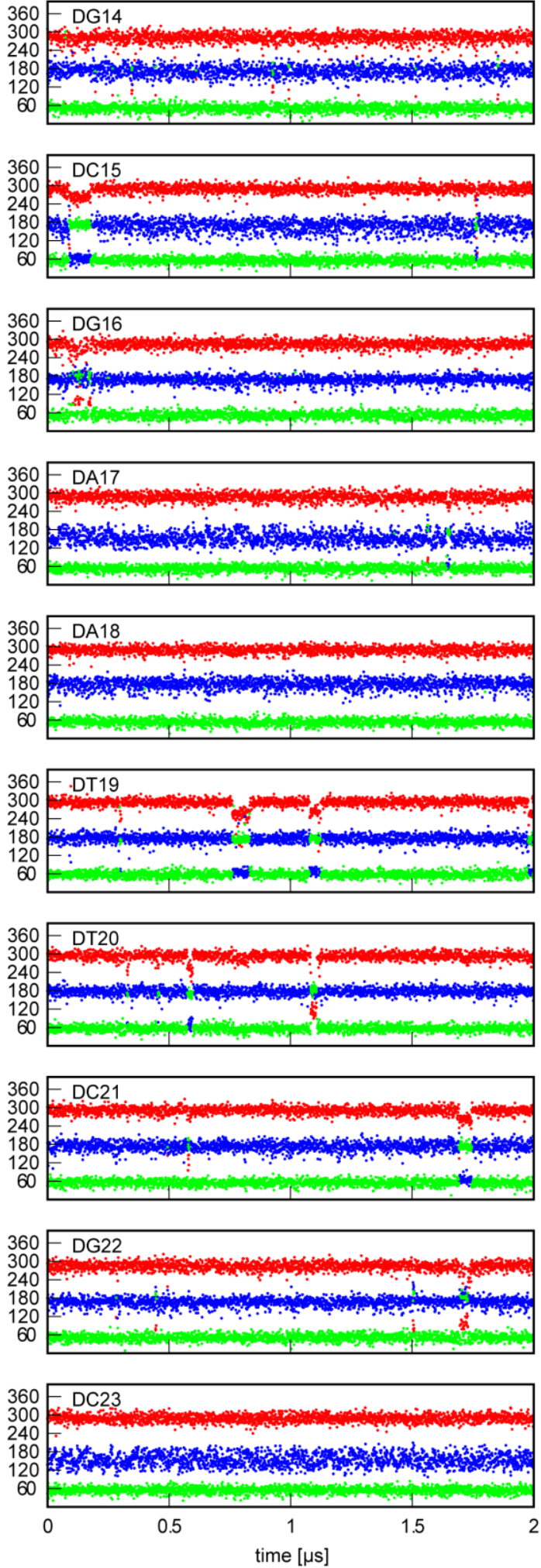
Figure S8. RMSD of two terminal base CG pairs of Dickerson-Drew dodecamer (end 1 and end 2) calculated for all atoms against the 1BNA structure (mass weighted). Unrestrained simulation in $0.15 \mathrm{M} \mathrm{KCl}$ and $\mathrm{SPC} / \mathrm{E}$ water.
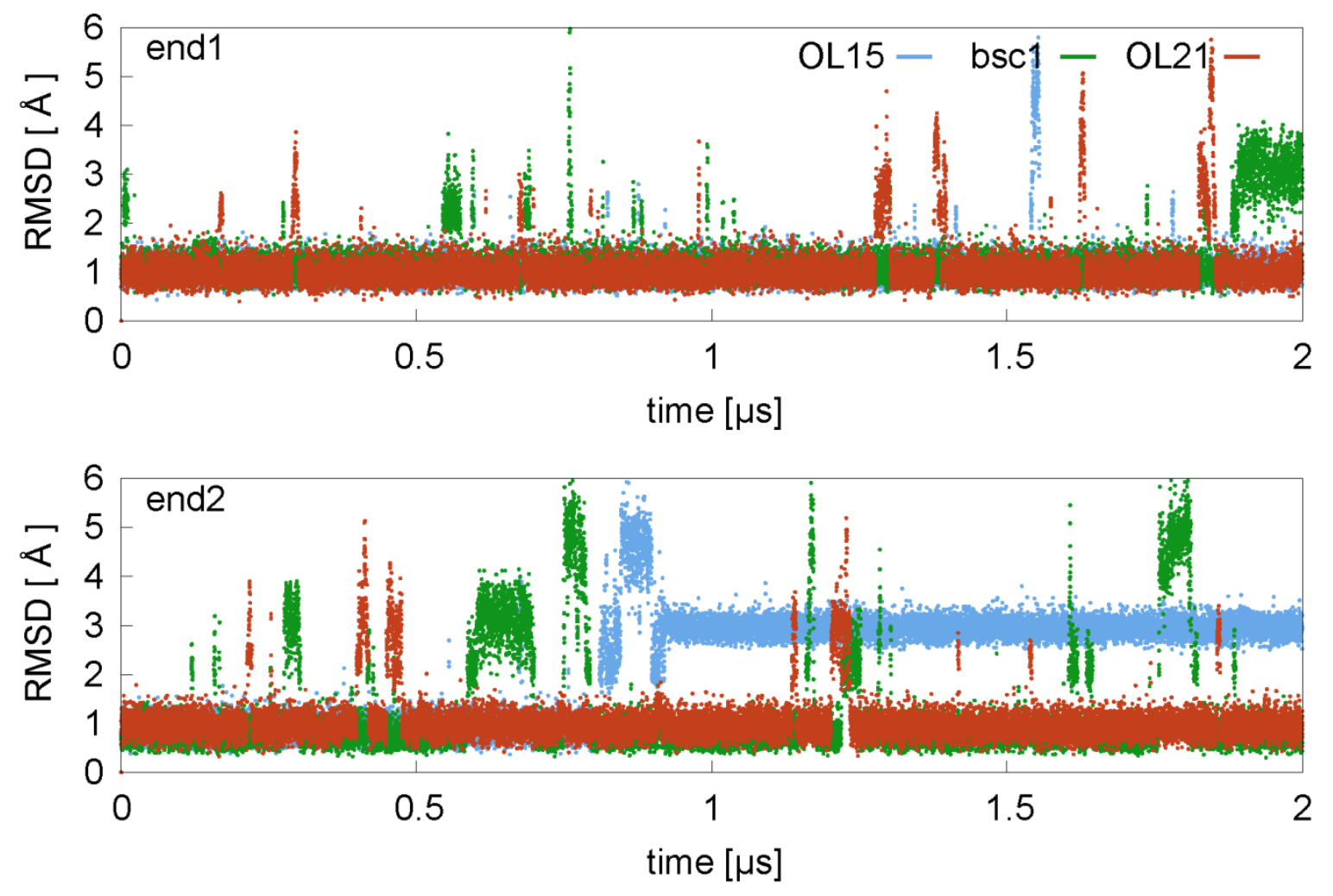

\section{Inclusion of conformation-dependent solvation effects and fitting $\alpha / \gamma$ parameters.}

Reference curves for fitting dihedral parameters were obtained as described in Ref 26 [Zgarbova et al., J Chem. Theory Comput. 2012, 8, 3232-3242] and below. In Eq. S1, $E_{\text {dih }}(\alpha, \gamma)$ is the $\alpha$ or $\gamma$ dihedral term to be fitted. E(QM/COSMO//QM/COSMO) are the QM single point energies calculated at the geometries optimized at the QM level with the COSMO solvent model. $\mathrm{E}(\mathrm{MM} / \mathrm{PB} / / \mathrm{MM} / \mathrm{PB})_{-\alpha, \gamma}$ are the $\mathrm{MM}$ single point energies calculated on $\mathrm{MM}$ optimized geometries, both with PB solvent model with (selected) $\alpha$ or $\gamma$ torsional terms set to zero $(-\alpha, \gamma)$.

$E_{d i h}(\alpha, \gamma)^{\text {solv }}=E(Q M / C O S M O / / Q M / C O S M O)-E(M M / P B / / M M / P B)_{-\alpha, \gamma}$

The selected dihedral force field terms for the $\alpha$ or $\gamma$ torsions set to zero in the second term on the right-hand side of Eq. S1 are: terms on the O3'-P-O5'-C5' atoms for the $\alpha$ dihedral and on the $\mathrm{O}^{\prime}-\mathrm{C5}^{\prime}-\mathrm{C}^{\prime}-\mathrm{C} 3^{\prime}$ atoms for the $\gamma$ dihedral. Original terms were kept on the O1P-P-O5'-H5' and 


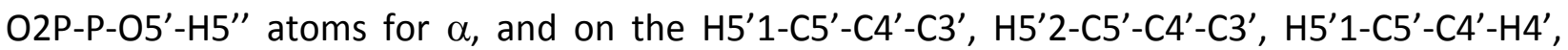
$\mathrm{H} 5^{\prime} 2-\mathrm{C}^{\prime}-\mathrm{C}^{\prime}-\mathrm{H} 4^{\prime}, \mathrm{H} 5^{\prime} 1-\mathrm{C}^{\prime}-\mathrm{C}^{\prime}-\mathrm{O} 4^{\prime}, \mathrm{H} 5^{\prime} 2-\mathrm{C}^{\prime}-\mathrm{C}^{\prime}-\mathrm{O}^{\prime}, \mathrm{O}^{\prime}-\mathrm{C}^{\prime}-\mathrm{C}^{\prime}-\mathrm{H} 4^{\prime}, \mathrm{O} 5^{\prime}-\mathrm{C}^{\prime}-\mathrm{C}^{\prime}-\mathrm{O} 4^{\prime}$ atoms for

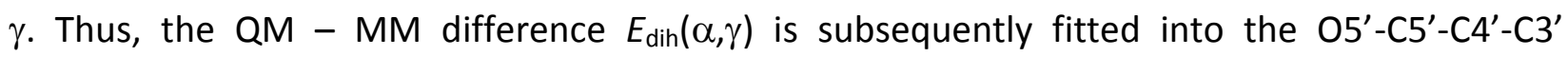
dihedral term for $\alpha$ torsion and $\mathrm{O} 5^{\prime}-\mathrm{C5}^{\prime}-\mathrm{C}^{\prime}-\mathrm{C} 3^{\prime}$ term for $\gamma$ torsion.

Note that in Eq. S1, QM (COSMO) and MM (PB) solvation energies are subtracted, thus the bulk of solvation energy is not fitted into the torsion parameters. However, conformation-dependent solvation effects, which are included in the QM COSMO calculation and not present in the MM PB calculation (MM molecule is not polarizable) are thus included in the parameterization.

\section{Effect of water model}

The ZI and ZII Z-DNA forms differ in the compactness of the charge arrangement in the GpC step. $\mathrm{Zl}$ is more compact in the sense that the phosphates neighbouring the phosphate of the $\mathrm{GpC}$ step are on average closer to that phosphate than in the ZII form, as shown in Table S3.

Table S3. Average P-P distances ( $\AA$ ) between the phosphate of the GpC step and its 5' - and 3'neighboring phosphates for ZI and ZII substates. OL21 simulation.

\begin{tabular}{|c|c|c|c|c|c|c|}
\hline \multirow{2}{*}{ Water } & \multicolumn{2}{|c|}{ P-P dist in $\mathrm{ZI}[\AA ̊]$} & \multicolumn{2}{|c|}{ P-P dist in ZII [Å] } & \multirow[t]{2}{*}{$\% \mathrm{ZI}$} & \multirow[t]{2}{*}{$\%$ ZII } \\
\hline & to $5^{\prime} \mathrm{P}$ & to $3^{\prime} \mathrm{P}$ & to $5^{\prime} \mathrm{P}$ & to $3^{\prime} \mathrm{P}$ & & \\
\hline SPC/E & 6.50 & 5.82 & 7.19 & 6.55 & 90 & 7 \\
\hline TIP3P & 6.53 & 5.77 & 7.20 & 6.50 & 85 & 7 \\
\hline OPC & 6.52 & 5.89 & 7.20 & 6.50 & 82 & 10 \\
\hline
\end{tabular}

The more concentrated negative charge of the $\mathrm{Zl}$ conformation requires better electrostatic screening, which comes largely from the cations present in solution ( $2 \mathrm{M} \mathrm{Na}^{+}$in our simulation). Thus, the $\mathrm{P} . . . \mathrm{Na}^{+}$radial distribution function (RDF) reflects how well the GpC step phosphate is shielded from neighbouring negative charges. RDFs for the GpC step phosphate and $\mathrm{Na}^{+}$in solution are shown in Figure S9 and their integrals in Figure S10. We can see that more sodium ions come closer to the GpC step phosphate in the SPC/E water than in the TIP3P and OPC water models (considering contact and solvent separated pair distances). Thus, P-P shielding (and stabilization of the ZI form) is probably more efficient in SPC/E, less so in TIP3P and even less in OPC water. This correlates with the population of the $\mathrm{Zl}$ conformation, which is highest in the SPC/E water due to the best shielding from the sodium ion atmosphere. However, it should be noted that the differences in the interaction of sodium ions with phosphate groups are the result of both the water model and sodium parameters, as different water models use different optimized sodium parameters. Thus, the resulting screening effect cannot be attributed solely to the water model, but rather to the combination of water and ion parameterization.

Because compactness of the negative phosphate charge does not significantly differ for the CpG step conclusive explanation could not be reached for this step and conformational equilibrium in it. 
Figure S9. RDF of GpC step phosphate and $\mathrm{Na}^{+}$in solution.
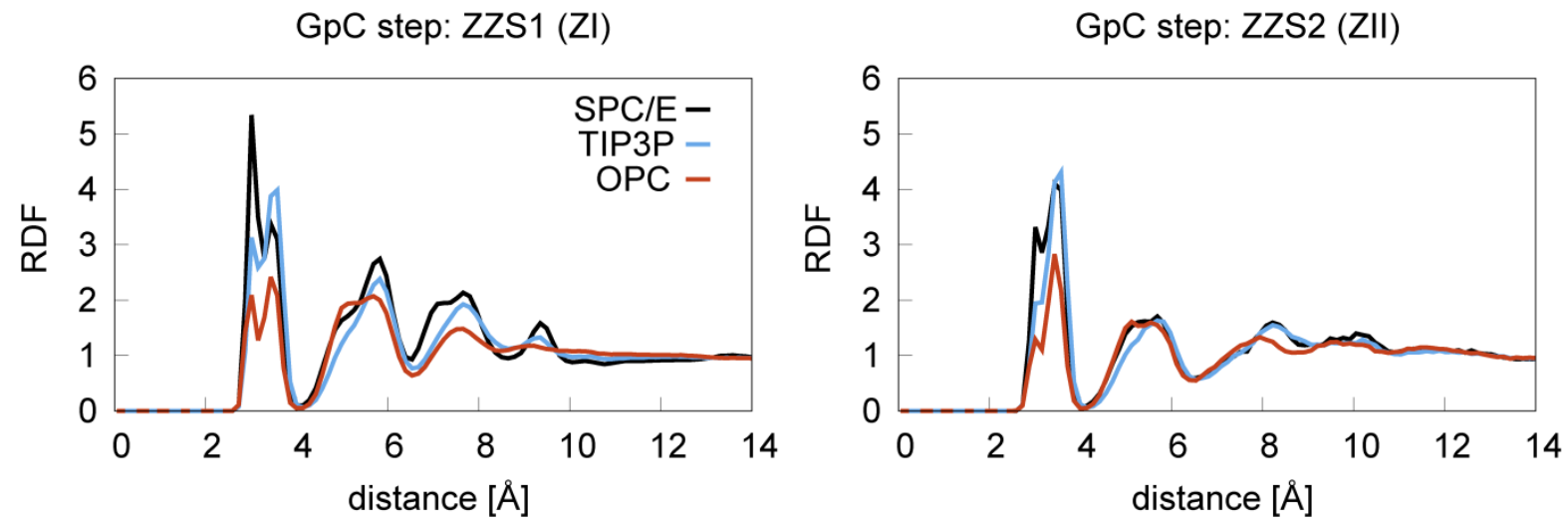

Figure S10. Integral of the RDFs of the GpC step phosphate and $\mathrm{Na}^{+}$in solution.
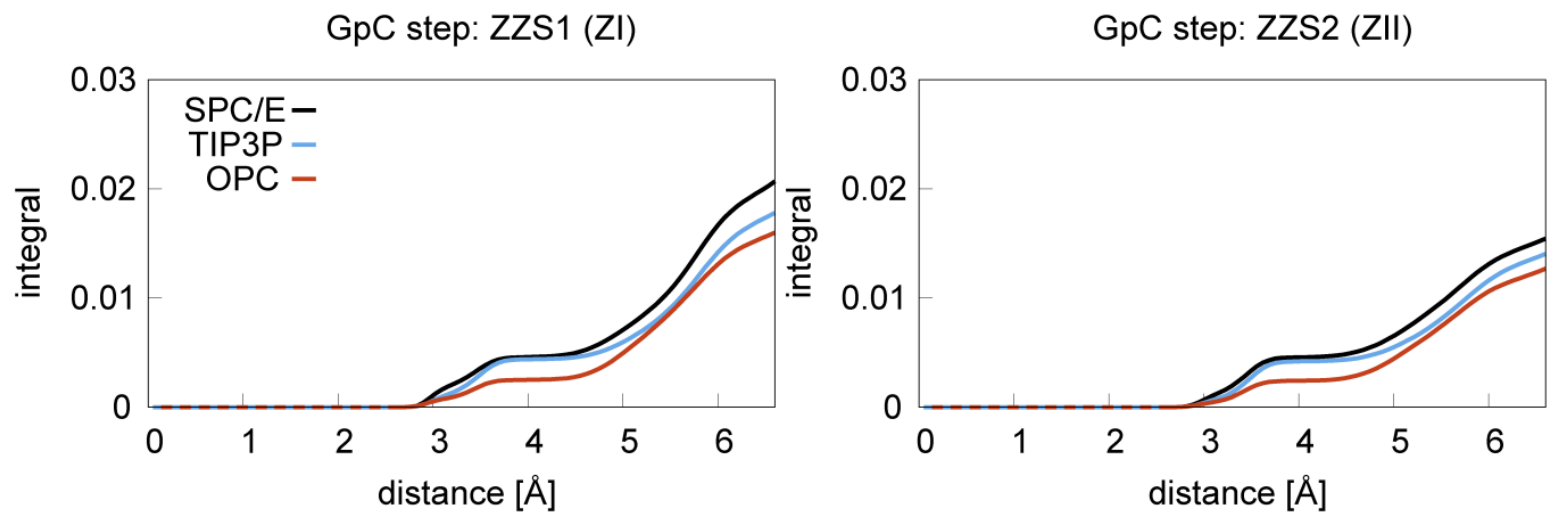